\title{
Prognostic role of tissue transglutaminase 2 in colon carcinoma
}

María Jesús Fernández-Aceñero ${ }^{1,2 q^{*}}$, Sofía Torres ${ }^{3 \pi}$, Irene Garcia-Palmero ${ }^{3}$, Cristina Díaz del Arco $^{1}$ and J. Ignacio Casal ${ }^{3 *}$

From the Departments of ${ }^{1}$ Surgical Pathology, Hospital Clínico San Carlos and ${ }^{2}$ Fundación Jiménez Díaz; and ${ }^{3}$ Cellular and Molecular Medicine. Centro de Investigaciones Biológicas (CIB) (Madrid, Spain).

Co-corresponding authors: Dra. MJ Fernández Aceñero, Department of Surgical Pathology. Hospital Clínico San Carlos, C/Prof. Martín Lagos s/n 28040 Madrid (Spain) and Dr. JI Casal Centro de Investigaciones Biológicas. CSIC. Madrid, Spain.

E-mail to: jmariajesus.fernandez@salud.madrid.org

The authors acknowledge no conflict of interest for the elaboration of the present report.

ๆ. These authors contributed equally to the manuscript

*. Co-corresponding authors 


\section{Abstract}

Tissue transglutaminase (TG2) is involved in a great number of biological processes, from wound healing to neurodegeneration. Recently, there has been an increasing interest in this enzyme as a potential prognostic marker or therapeutic target in human neoplasms. The aim of this study was to analyze mRNA and immunohistochemical expression of TG2 in colon cancer samples and to evaluate the potential prognostic value of this marker. We investigated not only increased expression but also differential location of the protein in the tumor in a clinical series of human tumors. "In silico" analysis using the GSE39582 dataset showed that TG2 expression was associated with an earlier relapse. The results of $\mathrm{qPCR}$ in our series showed that TG2 mRNA was upregulated in 25 out of 70 samples (34\%). Kaplan-Meier plots and the log-rank test showed that patients with increased TG2 mRNA expression showed significantly worse prognosis in terms of overall survival (OS) and a trend to earlier recurrence. Immunohistochemistry of tumor sections revealed stromal staining for TG2 in 152 cases (88\%) and epithelial cell staining in 105 cases (62\%). Stromal expression in stage II patients showed a significant association to DFS. Association with poor prognosis was also confirmed for patients with metastatic disease. A Cox multivariate analysis showed that TG2 expression in epithelial cells behaved as a significant independent prognosticator of OS, together with node involvement and presence of metastasis, while stromal TG2 expression was associated to DFS. In summary, stromal TG2 expression was significantly associated to DFS in non-metastatic patients and epithelial TG2 expression was significantly associated to OS, independently of node involvement and metastasis.

Keywords: Transglutaminase 2; colon carcinoma; prognosis; mRNA; metastasis 


\section{Introduction:}

Transglutaminase 2 (TG2) is a member of the transglutaminase family, also called tissue transglutaminase. TG2, encoded by a stress-responsive gene, is a transcriptional target of hypoxia-inducible factor (HIF-1 $\alpha$ ) [1]. TG2 is a calcium-dependent multifunctional protein-crosslinking enzyme and a dual function G-protein [2]. Although, the diverse biological activities of TG2 are mainly modulated by calcium, other factors like GTP or the redox potential can regulate its function [3].

TG2 is up-regulated in wound healing [4-7] and has been implicated in the pathogenesis associated to fibrogenic reactions in various organs [8]. It catalyzes the crosslinking of a wide variety of extracellular matrix proteins for regulation of extracellular matrix (ECM) stability and reconstruction of intracellular cytoskeleton [9]. TG2 associates with some members of the integrin family to promote stable interactions between cells and the ECM, resulting in increased cell survival, migration, and invasion [10-12]. Actually, TG2 could be involved in signal transduction and transformation via microvesicles.

Besides, it has been found highly expressed in tumor tissues of various origins $[7,8,10-13]$, associated with therapy resistance [14] and metastatic behavior $[3,15]$. TG2 is involved in tumor progression through the regulation of multiple biological processes such as cell adhesion, motility, invasion, survival and apoptosis [2]. TG2 can promote invasive ability of malignant cells by modulating the ECM and the epithelialmesenchymal transition (EMT) [16-18]. The mechanisms involved in TG2 oncogenic potential are not fully understood. Some reports have linked them to the activation of nuclear factor kappa $\beta$ (NFK $\beta$ ), focal adhesion kinase (FAK) [19] or to the dowregulation of the tumor suppressor PTEN [20]. Recently, an association between TG2 expression and the increase of transcriptional repressors Snail1, Twist, Zeb1 and 
Zeb2 has been found in mammary tumors cells. This mechanism promotes EMT and enhances metastatic behavior of tumor cells [18, 21, 22]. In addition, its expression is linked with activation of pathways that are known to increase cancer stem cells $[23,24]$.

Recently, it has been described that TG2 could be expressed both in tumor epithelial cells [24] as in the tumor stroma [25-28]. In breast cancer, stromal TG2 expression was inversely correlated with disease free survival of patients [26]. Park et al. showed that strong TG2 epithelial expression was significantly associated with poor prognosis in renal cell carcinoma [29]. In colorectal cancer, high TG2 mRNA expression was a marker of bad prognosis in a series of 91 patients with colon cancer [30]. In contrast, TG2 downregulation in SW colon cancer cell lines induced higher invasiveness and aggressiveness [31]. Indeed a lower expression of TG2 in liver metastasis was reported [31]. However, no human sample series have been used to analyze the prognostic influence of the cellular location of TG2 expression by immunohistochemistry. Differential location of TG2 may have different clinical implications according to the tumor type and stage. Here, our aim was to determine the clinical relevance of stromal and epithelial TG2 overexpression in a retrospective series of patients with colon carcinoma. 


\section{Patients and Methods}

\section{Study design}

We used a retrospective case series of colon carcinoma patients. Before study initiation we performed a sample size analysis. By selecting 200 patients we could obtain a $90 \%$ power with an alpha error of 0.05 as conventionally accepted.

All the patients enrolled in the present study were diagnosed and treated at Fundación Jiménez Díaz Hospital (Madrid, Spain) and followed up for at least 5 years. We collected a retrospective case series of 198 patients with colon carcinoma to evaluate the prognostic influence of TG2 expression (Supplementary Table S1). Colon carcinoma cases were randomly selected from those diagnosed in our Hospital between January 2003 and December 2009. As rectal carcinoma patients receive neoadjuvant therapy before surgery, we excluded these patients from our study. Demographic features have been retrieved from the electronic files of the patients. Patients with TNM stages I and II did not receive further therapy after surgery, while stages III and IV received adjuvant chemotherapy, based in oxaliplatin and 5-fluorouracil. Informed written consent was obtained from all participants, as required and approved by the Research Ethics Committee of the Hospital.

The main outcome variables of the study were disease free survival (DFS), defined as the time period elapsed between surgical therapy of the primary tumor and disease relapse (in months), and overall survival (OS), defined as the time period elapsed between primary therapy and death of disease (also in months). For DFS we analyzed cases at stages I, II and III, and for OS analysis the whole cohort. 


\section{External dataset}

For in silico analysis, we used the GSE39582 dataset, which contains a cohort of 585 patients of colon cancer. Data were transformed in z-scores and z-ratios values. Positive and negative z-ratios indicated a significantly higher or lower expression level.

\section{Tissue samples}

All the tissue samples from the 198 patients were obtained from the Surgical Pathology Department (Fundación Jiménez Díaz, Madrid) and reviewed by two pathologists specialized in Gastrointestinal Pathology to confirm pathological stage and grade of the tumors. For quantitative PCR, we prepared RNA from 70 paired samples from whole sections of formalin-fixed paraffin-embedded tissue. Then, we constructed a tissue microarray (TMA) with two $1 \mathrm{~mm}$ cores from each patient to get a wide representation of the whole tumor. The TMA consisted of 172 colon cancer and 49 liver metastasis (Supplementary Table S1).

\section{Quantitative PCR}

We used whole sections containing more than $85 \%$ of tumor, but we did not perform macro or microdissection for mRNA extraction. RNA was isolated from 3x10 $\mu$ msections from formalin-fixed paraffin-embedded tissue using the "NucleoSpin totalRNA FFPE XS” kit (Macherey-Nagel). cDNA was subsequently obtained using SuperScript II First Strand Synthesis System with random hexamers (Invitrogen). Real-time quantitative PCR (QPCR) was performed using the FastStart Master Mix (Roche) with probes from the Universal Probe Library Set (Roche). Amplifications were run in a LightCycler® 480 (Roche). Each value was adjusted using $\beta$-actin and 18S RNA levels as references. Expression of the selected genes was considered positive when the tumor/normal ratio showed a value $\geq 2$. 


\section{Immunohistochemistry}

To confirm protein location within the tumor, we performed TG2 immunohistochemistry in tissue samples. Each sample was deparaffinized for antigen retrieval (microwaving) using sodium citrate ( $\mathrm{pH}$ 6.0) for $20 \mathrm{~min}$ and subsequently incubated with TG2 antibody (1:250; ab73170; Abcam). We used EnVision Dual Link system-HRP (Dako), this system is based on an horseradish peroxidase labelled polymer which is conjugated with secondary antibodies. The reaction was developed using diaminobenzidine (DAB) as chromogen and hematoxylin for counterstaining. In all cases, sections from normal colonic mucosa distant from the tumor site were used as negative controls.

All the slides were reviewed independently by two pathologists, blinded to the outcome of the patients. Immunohistochemistry was scored with a quickscore scale. For this aim we measured the percentage of cells showing cytoplasmic and/or membrane staining and the intensity of the expression (weak $[=1]$, moderate $[=2]$ or intense $[=3]$ ) and calculated a $\mathrm{z}$ score (intensity $\mathrm{x}$ percentage, values ranging from 0-300). Subsequently we built a ROC curve model to define the best cut-off point and to classify cases as negative or positive. In both cases, epithelial and stromal, the cut-off point was settled at 150 , meaning $50 \%$ of cells with intense reaction or more than $75 \%$ with moderate intensity, being sub-classified as negative $(<150)$, moderate $(151-225)$ or intense ( $>225)$. Cases with weaker expression of the marker were considered negative. The concordance rate for these measures was over $80 \%$. Discordant cases were cojointly reviewed to achieve agreement.

\section{Statistical analysis}


All the variables of the study have been described using mean (standard deviation) or percentages, as required. Associations between variables were analyzed with the Chisquared or Student's t test for mean comparison. The univariate survival analysis was investigated using the Kaplan-Meier plots and compared by the log-rank test. We adjusted a Cox multivariate survival model for DFS and OS. The significance was established at $\mathrm{p}<0.05$ for all tests, except survival $(\mathrm{p}<0.1)$, which was considered a trend to significance. All the analyses were performed with SPSS 20.0 for Windows. 


\section{Results}

\section{Prognostic value of TG2 mRNA expression in colon cancer}

To assess the capacity of TG2 expression for the outcome prediction of colon cancer patients, we used first the external public dataset GSE39582 (585 patients) and then qPCR. For "in silico" analysis, patients were divided either into "low" expression (negative z-score) or "high" expression (positive score). Data were plotted using Kaplan Meier survival curves and the prognostic value was tested with log rank analysis and univariate Cox proportional hazards models. Patients without metastasis at diagnosis and high expression of TG2 showed earlier relapse than patients with lower expression levels (HR 1.59 [95\% CI: 1.2-2.3]; log-rank test $\mathrm{p}$ value=0.006) (Figure 1A), but no significant association was found to OS (Figure 1B). Interestingly, stage II patients showed shorter time to recurrence when TG2 was highly expressed (HR 2.38 [95\% CI: 1.4-4.1]; $\mathrm{p}$ value $=0.001)($ Figure $1 \mathrm{C})$.

Then, we analyzed TG2 mRNA expression in 70 paired colon cancer samples using qPCR (Supplementary Table S2). Females were slightly over-represented compared to males, but cancer location was uniformly distributed between right and left colon, reflecting the most usual clinical scenario. Most cases were stage II or III, although we have a significant proportion of patients with metastatic disease at diagnosis (stage IV). In these patients, 25 out of 70 (34\%) showed high TG2 expression. No significant association was found between TG2 expression and clinicopathological parameters (Supplementary Table S3). However, Kaplan-Meier analysis showed a statistically significant association between high TG2 mRNA expression and OS for the whole series (HR 4.3 [95\% CI: $1.4-12$ ]; $\mathrm{p}$ value $=0.008)$ and a trend to significance for DFS in stage I-III patients (HR 2.1 [95\% CI: 0.98-4.5]; $\mathrm{p}$ value $=0.057$ ) (Figure 1D-E). 
Therefore, high TG2 mRNA expression was significantly associated to a worse outcome. The discrepancy between "in silico" analysis and qPCR could be explained by the criteria used to assign patients to the "high" and "low" expression categories. In silico analysis does not consider the differences in the normal levels for each patient and the cut-off is determined according to the median expression of tumors, while for our analysis we have considered a positive value when the tumor/normal ratio showed a value $\geq 2$

\section{Prognostic value of TG2 expression according to its location}

Use of whole tissue section without micro o macrodissection for mRNA extraction does not allow the distinction between stromal and epithelial expression. To study the precise location of TG2 within the tumor we performed immunohistochemistry using a TMA consisting of 172 patients with colon carcinoma (Supplementary Table S4). IHC analysis showed staining of the stromal cells in $89 \%$ of the patients, as opposed to $61 \%$ epithelial cells exhibiting TG2 expression (Figure 2A). To note, we did not find significant association between stromal and epithelial expression of TG2 (likelihood ratio $\mathrm{p}$ value $=0.6$ ). Stromal staining was intense/ moderate while the epithelial staining was mainly moderate, with intense staining in $11 \%$ of the samples (Figure $2 \mathrm{~B}$ ).

To investigate the effect of differential location, TG2 expression was analyzed by $\mathrm{qPCR}$ and IHC in 49 patients. 14 patients out of $16(87.5 \%)$ with high level of expression mRNA of TG2, were co-positive by immunohistochemistry and qPCR,

while 15 out of 33 patients $(46 \%)$ were co-negative in both techniques. Therefore, 18 out 33 patients (54\%) with low mRNA expression showed clear positivity using IHC. The conflicting results between $\mathrm{qPCR}$ and IHC might be explained due to the 
differential TG2 expression location observed in whole tissue and the different readouts for both techniques.

In the univariate statistical analysis we found a significant association between TG2 epithelial expression, the presence of metastasis at diagnosis and the TNM stage. We observed that low TG2 expression was associated to more advanced disease. Left and sigmoid colon tumors together exhibited significantly higher TG2 stromal expression when compared to cecum, right and transverse colon lesions (Supplementary Tables S5-6). No other significant associations for TG2 were found for stromal expression.

Whereas Kaplan Meier survival analysis did not find a significant association for TG2 epithelial expression (Figure 3A, B), TG2 stromal expression was associated to DFS in non-metastatic patients (Figure $3 \mathrm{C}$, D). To test whether intensity influenced prognosis or not, we performed another analysis of stromal expression dividing positive cases in moderate or intense. Patients with intense stromal staining showed shorter time to recurrence than patients with negative expression. Moderate staining patients presented an intermediate risk of relapse (Figure 3D), including patients at stage II (Figure 3E). In addition, a significant association between TG2 staining in epithelial cells and overall survival was found in patients with metastasis at the time of diagnosis (stage IV disease) (Figure 3F). Then, we performed a Cox multivariate survival model to define those factors significantly and independently associated with survival and relapse. A significant and independent association between epithelial TG2 expression and OS was found (Supplementary Table S7), while for stromal expression, we only observed a trend towards significance for the association with DFS $(p<0.1)$ (Supplementary Table S8). 


\section{TG2 expression in liver metastasis}

Stage I-III patients with recurrence at 36 months exhibited higher TG2 stromal expression than patients with a better outcome $(60 \%$ vs. $43 \%)(\mathrm{p}<0.1)$ (Figure 4A). However, no association was found between recurrence and epithelial expression (Figure 4B). Then we tested TG2 expression in 49 liver metastasis. Analysis of the association between TG2 expression in stromal cells of the primary tumor and the corresponding metastasis showed no significant changes of the stromal expression in the metastasis $(\mathrm{p}=0.3)$. Significant association was neither noted for epithelial expression, but 13 out of 20 metastasis lost TG2 expression in positive primary tumors. Stromal expression was higher (85\% of patients) than epithelial expression (29\%) (Figure 4C). Besides, IHC staining showed that epithelial expression was lower in liver metastasis than in primary colon cancer (29\% vs. $61 \%$ of patients) (Figure 4D). TG2 stromal expression was higher in patients with liver metastasis, suggesting that TG2 expression in colon cancer-associated fibroblasts might be involved in tumor progression and metastasis. 


\section{Discussion}

In this study, we have analyzed the prognostic value of TG2 in colon cancer using "in silico" analysis, qPCR and immunohistochemistry combined with the first clinical series to study the prognostic influence of both techniques. To our knowledge, this is the first study to determine the independent prognostic value of stromal and epithelium expression of TG2 in colon carcinoma. We found that $89 \%$ of patients expressed TG2 in stromal compartment, for $61 \%$ in tumor epithelium. Stromal expression allowed stratifying the risk of relapse, even at stage II. In this group of patients adjuvant therapy is not usually recommended, although almost $20 \%$ of cases can relapse and even die of disease. Therefore, it is especially interesting to define factors predicting poor outcome to decide further therapeutic alternatives after surgery.

Epithelial expression seemed to be less frequent in metastatic cases, but those patients with stage IV patients and high TG2 expression in metastatic epithelium showed a significantly shorter survival time. Our data help to understand previous apparently conflicting reports [30, 31]. TG2 loss of expression in liver metastasis is compatible with the association of TG2 high expression to worse prognosis found in stage IV patients in the present series. Although only a small percentage of metastatic patients express epithelial TG2, its high expression correlates with worse outcome. On the other hand, overall high TG2 expression correlates with DFS and OS as we have observed.

TG2 showed a prognostic value by qPCR in DFS as well as in OS. However, the in silico analysis showed that non-metastatic patients with high TG2 expression displayed earlier relapses of the disease, but no association was found with OS. This discrepancy may be due to several causes. Patients are categorized differently as high or 
low expression in both studies (in silico analysis by the median expression while analysis of mRNA fold-change expression is relative to adjacent normal tissue). By immunohistochemical analysis, the majority of patients exhibited increased TG2 expression in the stromal compartment whereas $61 \%$ of patients exhibited a moderate epithelial expression. Stromal expression showed inverse correlation to DFS while epithelial expression was associated to OS in patients with metastases at diagnosis. We have observed a good correlation between positive cases for qPCR and IHC analysis. Some discrepancies between both tests might be due to the fact that qPCR is performed on whole tissue sections and the presence of multiple cell types in the tumor could affect the final results.

During last years, many evidences have been reported indicating the role of tumor stroma in the development and progression of tumors [25, 32]. TG2 expression increased in colon cancer associated fibroblasts (CAFs) compared to normal fibroblasts [25] and CAFs acquired a phenotype similar to myofibroblasts in wound healing, where TG2 is implicated and up-regulated [4-7]. In fact, fibroblasts overexpressing TG2 showed high adhesion and better extensibility [4]. On the other hand, microvesicles containing TG2 shed by cancer cells are capable of conferring the transformed characteristics of cancer cells onto normal fibroblasts and epithelial cells [33]. TG2 is up-regulated by TGF $\beta$ and promotes incorporation and activation of TGF $\beta$ into matrix $[2,34]$, which might explain the increased TG2 expression in tumor stroma, and its association with increased risk of relapse in non-metastatic patients. In addition, we have observed high stromal expression levels of TG2 in metastatic livers, similar to the TG2 stromal expression in primary tumors. Therefore, TG2 expression in the tumor stroma seems to be necessary for progression into metastasis in colon cancer. 
TG2 induces chemoresistance through the inhibition of apoptosis and autophagy, induction of EMT and stemness. [18,21,23] Another interesting aspect of TG2 is its use as a potential therapeutic target. TG2 siRNA has been successfully used to reverse drug resistance in preclinical models of ovarian and pancreatic carcinomas, but the clinical evidence is still scarce and further studies are needed $[35,36]$. A good alternative could be the development of small molecule inhibitors selectively targeting TG2. This kind of drugs has already been employed in some inflammatory disorders with some promise [37]. In mammary tumor cell lines, inactive TG2 is still able to effectively induce EMT and drug resistance through the activation of NF- $\mathrm{BB}$ and other pathways [38]. Therefore, it might be better to identify molecules that can bind TG2 and lock it in an inactive state regardless of enzymatic activity [39]. Interestingly, the antioncogenic action of cantharidine was associated to a reduction of TG2 in human tumor tissue samples [40].

In conclusion, our results indicate $\mathrm{TG} 2$ expression is a marker of poor outcome in colon carcinoma. Prognostic value is associated to the differential location of TG2 expression. Stromal expression allows stratifying the relapse risk, while epithelial expression in metastatic patients is associated with poor overall survival. Therefore, TG2 expression can help to take medical decisions in the management of colon cancer patients.

\section{ACKNOWLEDGEMENTS}


Compliance with Ethical Standards: This work is in accordance with national and international Ethical Standards and has been approved by the Ethical Committee of Hospital Clínico San Carlos and Fundación Jiménez Díaz.

Funding: ST was a recipient of the Juan de la Cierva programme. IGP was supported by a contract S2010/BMD-2344/Colomics2 from the Comunidad de Madrid. This research was supported by grants BIO2012-31023 and BIO2015- 66489-R from the MINECO and PRB2 (IPT13/0001-ISCIII-SGEFI/FEDER) from the Instituto de Salud Carlos IIIFEDER.

Conflict of Interest: The authors declare no conflict of interest for the present study 


\section{References:}

1. Filiano AJ, Bailey CD, Tucholski J, Gundemir S, Johnson GV (2008) Transglutaminase 2 protects against ischemic insult, interacts with HIF1beta, and attenuates HIF1 signaling FASEB J 22:2662-2675

2. Huang L, Xu AM, Liu W (2015) Transglutaminase 2 in cancer Am J Cancer Res 5:27562776

3. Agnihotri N, Kumar S, Mehta K (2013) Tissue transglutaminase as a central mediator in inflammation-induced progression of breast cancer Breast Cancer Res 15:202

4. Stephens $P$, Grenard $P$, Aeschlimann P, Langley M, Blain E, Errington R, Kipling D, Thomas D, Aeschlimann D (2004) Crosslinking and G-protein functions of transglutaminase 2 contribute differentially to fibroblast wound healing responses J Cell Sci 117:3389-3403

5. Wang Z, Collighan RJ, Gross SR, Danen EH, Orend G, Telci D, Griffin M (2010) RGDindependent cell adhesion via a tissue transglutaminase-fibronectin matrix promotes fibronectin fibril deposition and requires syndecan-4/2 alpha5beta1 integrin cosignaling J Biol Chem 285:40212-40229

6. Mehta K (2005) Mammalian transglutaminases: a family portrait Prog Exp Tumor Res 38:1-18

7. Mehta K, Kumar A, Kim HI (2010) Transglutaminase 2: a multi-tasking protein in the complex circuitry of inflammation and cancer Biochem Pharmacol 80:1921-1929

8. Aeschlimann D, Thomazy V (2000) Protein crosslinking in assembly and remodelling of extracellular matrices: the role of transglutaminases Connect Tissue Res 41:1-27

9. Telci D, Griffin M (2006) Tissue transglutaminase (TG2)--a wound response enzyme Front Biosci 11:867-882

10. Chen SH, Lin CY, Lee LT, Chang GD, Lee PP, Hung CC, Kao WT, Tsai PH, Schally AV, Hwang JJ, Lee MT (2010) Up-regulation of fibronectin and tissue transglutaminase promotes cell invasion involving increased association with integrin and MMP expression in A431 cells Anticancer Res 30:4177-4186

11. Wang Z, Griffin M (2013) The role of TG2 in regulating S100A4-mediated mammary tumour cell migration PLoS One 8:e57017

12. Erdem M, Erdem S, Sanli O, Sak H, Kilicaslan I, Sahin F, Telci D (2014) Up-regulation of TGM2 with ITGB1 and SDC4 is important in the development and metastasis of renal cell carcinoma Urol Oncol 32:25 e13-20

13. Chhabra A, Verma A, Mehta K (2009) Tissue transglutaminase promotes or suppresses tumors depending on cell context Anticancer Res 29:1909-1919

14. Ai L, Kim WJ, Demircan B, Dyer LM, Bray KJ, Skehan RR, Massoll NA, Brown KD (2008) The transglutaminase 2 gene (TGM2), a potential molecular marker for chemotherapeutic drug sensitivity, is epigenetically silenced in breast cancer Carcinogenesis 29:510-518

15. Mangala LS, Fok JY, Zorrilla-Calancha IR, Verma A, Mehta K (2007) Tissue transglutaminase expression promotes cell attachment, invasion and survival in breast cancer cells Oncogene 26:2459-2470

16. Cao L, Shao M, Schilder J, Guise T, Mohammad KS, Matei D (2012) Tissue transglutaminase links TGF-beta, epithelial to mesenchymal transition and a stem cell phenotype in ovarian cancer Oncogene 31:2521-2534

17. Giordano A, Gao H, Anfossi S, Cohen E, Mego M, Lee BN, Tin S, De Laurentiis M, Parker CA, Alvarez RH, Valero V, Ueno NT, De Placido S, Mani SA, Esteva FJ, Cristofanilli M, Reuben JM (2012) Epithelial-mesenchymal transition and stem cell markers in patients with HER2-positive metastatic breast cancer Mol Cancer Ther 11:2526-2534 
18. Kumar A, Xu J, Brady S, Gao H, Yu D, Reuben J, Mehta K (2010) Tissue transglutaminase promotes drug resistance and invasion by inducing mesenchymal transition in mammary epithelial cells PLoS One 5:e13390

19. Kim SY (2011) Transglutaminase 2: a new paradigm for NF-kappaB involvement in disease Adv Enzymol Relat Areas Mol Biol 78:161-195

20. Wang $Y$, Ande SR, Mishra S (2012) Phosphorylation of transglutaminase 2 (TG2) at serine-216 has a role in TG2 mediated activation of nuclear factor-kappa B and in the downregulation of PTEN BMC Cancer 12:277

21. Antonyak MA, Miller AM, Jansen JM, Boehm JE, Balkman CE, Wakshlag JJ, Page RL, Cerione RA (2004) Augmentation of tissue transglutaminase expression and activation by epidermal growth factor inhibit doxorubicin-induced apoptosis in human breast cancer cells J Biol Chem 279:41461-41467

22. Kim Y, Eom S, Kim K, Lee YS, Choe J, Hahn JH, Lee H, Kim YM, Ha KS, Ro JY, Jeoung D (2010) Transglutaminase II interacts with rac1, regulates production of reactive oxygen species, expression of snail, secretion of Th2 cytokines and mediates in vitro and in vivo allergic inflammation Mol Immunol 47:1010-1022

23. Calcagno AM, Salcido CD, Gillet JP, Wu CP, Fostel JM, Mumau MD, Gottesman MM, Varticovski L, Ambudkar SV (2010) Prolonged drug selection of breast cancer cells and enrichment of cancer stem cell characteristics J Natl Cancer Inst 102:1637-1652

24. Kumar A, Gao H, Xu J, Reuben J, Yu D, Mehta K (2011) Evidence that aberrant expression of tissue transglutaminase promotes stem cell characteristics in mammary epithelial cells PLoS One 6:e20701

25. Torres S, Garcia-Palmero I, Herrera M, Bartolome RA, Pena C, Fernandez-Acenero MJ, Padilla G, Pelaez-Garcia A, Lopez-Lucendo M, Rodriguez-Merlo R, Garcia de Herreros A, Bonilla F, Casal JI (2015) LOXL2 Is Highly Expressed in Cancer-Associated Fibroblasts and Associates to Poor Colon Cancer Survival Clin Cancer Res 21:4892-4902

26. Assi J, Srivastava G, Matta A, Chang MC, Walfish PG, Ralhan R (2013) Transglutaminase 2 overexpression in tumor stroma identifies invasive ductal carcinomas of breast at high risk of recurrence PLoS One 8:e74437

27. Singer CF, Hudelist G, Walter I, Rueckliniger E, Czerwenka K, Kubista E, Huber AV (2006) Tissue array-based expression of transglutaminase-2 in human breast and ovarian cancer Clin Exp Metastasis 23:33-39

28. Leicht DT, Kausar T, Wang Z, Ferrer-Torres D, Wang TD, Thomas DG, Lin J, Chang AC, Lin L, Beer DG (2014) TGM2: a cell surface marker in esophageal adenocarcinomas J Thorac Oncol 9:872-881

29. Park MJ, Baek HW, Rhee YY, Lee C, Park JW, Kim HW, Moon KC (2015) Transglutaminase 2 expression and its prognostic significance in clear cell renal cell carcinoma J Pathol Transl Med 49:37-43

30. Miyoshi N, Ishii H, Mimori K, Tanaka F, Hitora T, Tei M, Sekimoto M, Doki Y, Mori M (2010) TGM2 is a novel marker for prognosis and therapeutic target in colorectal cancer Ann Surg Oncol 17:967-972

31. Cellura D, Pickard K, Quaratino S, Parker H, Strefford JC, Thomas GJ, Mitter R, Mirnezami AH, Peake NJ (2015) miR-19-Mediated Inhibition of Transglutaminase-2 Leads to Enhanced Invasion and Metastasis in Colorectal Cancer Mol Cancer Res 13:1095-1105

32. Torres S, Bartolome RA, Mendes M, Barderas R, Fernandez-Acenero MJ, Pelaez-Garcia A, Pena C, Lopez-Lucendo M, Villar-Vazquez R, de Herreros AG, Bonilla F, Casal JI (2013) Proteome profiling of cancer-associated fibroblasts identifies novel proinflammatory signatures and prognostic markers for colorectal cancer Clin Cancer Res 19:6006-6019

33. Antonyak MA, Li B, Boroughs LK, Johnson JL, Druso JE, Bryant KL, Holowka DA, Cerione RA (2011) Cancer cell-derived microvesicles induce transformation by transferring 
tissue transglutaminase and fibronectin to recipient cells Proc Natl Acad Sci U S A 108:4852-4857

34. Verderio E, Gaudry C, Gross S, Smith C, Downes S, Griffin M (1999) Regulation of cell surface tissue transglutaminase: effects on matrix storage of latent transforming growth factor-beta binding protein-1 J Histochem Cytochem 47:1417-1432

35. Hwang JY, Mangala LS, Fok JY, Lin YG, Merritt WM, Spannuth WA, Nick AM, Fiterman DJ, Vivas-Mejia PE, Deavers MT, Coleman RL, Lopez-Berestein G, Mehta K, Sood AK (2008) Clinical and biological significance of tissue transglutaminase in ovarian carcinoma Cancer Res 68:5849-5858

36. Verma A, Guha S, Diagaradjane $P$, Kunnumakkara AB, Sanguino AM, Lopez-Berestein $G$, Sood AK, Aggarwal BB, Krishnan S, Gelovani JG, Mehta K (2008) Therapeutic significance of elevated tissue transglutaminase expression in pancreatic cancer Clin Cancer Res 14:2476-2483

37. Badarau E, Collighan RJ, Griffin M (2013) Recent advances in the development of tissue transglutaminase (TG2) inhibitors Amino Acids 44:119-127

38. Budillon A, Carbone C, Di Gennaro E (2013) Tissue transglutaminase: a new target to reverse cancer drug resistance Amino Acids 44:63-72

39. Pinkas DM, Strop P, Brunger AT, Khosla C (2007) Transglutaminase 2 undergoes a large conformational change upon activation PLoS Biol 5:e327

40. Yang LL, Liang CY, Lu TC, Zhi CY, Liu B, Zhou JH, Liu XM, Gao HC, Huang W (2013) Role of tissue transglutaminase and effect of cantharidinate in human colorectal cancer Mol Med Rep 8:1812-1816 


\section{LEGENDS TO THE FIGURES}

Figure 1. Prognostic value of TG2 mRNA expression in colon cancer. Kaplan-Meier survival curves for OS and DFS of cases with high and low expression of TG2 mRNA in (A-C) 585 patients from the microarray GSE39582 and (D-E) QPCR analysis in a different cohort of 70 colon cancer patients. Kaplan-Meier curves were compared with the log-rank test and Hazard Ratio values were based in Cox's univariate survival model.

Figure 2. TG2 expression is mainly stromal in primary tumors of colon cancer patients. A) Examples of epithelial and stromal staining for TG2 in human colon cancer (TG2 IHC staining x200). B) Percentage of patients with negative, moderate or intense staining for TG2 in tumoral epithelium and stroma are shown.

Figure 3. TG2 stromal staining is associated with risk of recurrence in stage I-III patients. A-F) Kaplan-Meier curves for OS and DFS of patients with positive and negative TG2 staining for epithelial and stromal expression. Kaplan-Meier curves were compared with the log-rank test. The Hazard Ratio was based on Cox's univariate survival model.

Figure 4. Expression of TG2 in liver metastasis. A-B) Percentage of patients with recurrence at 36 months showing stromal or epithelial TG2 expression in primary tumors. C) Percentage of patients with negative, moderate or intense TG2 staining in liver metastasis. D) Examples of TG2 epithelial and stromal staining in human liver metastasis and paired primary colon samples in 44 cases (TG2 IHC staining x200). 


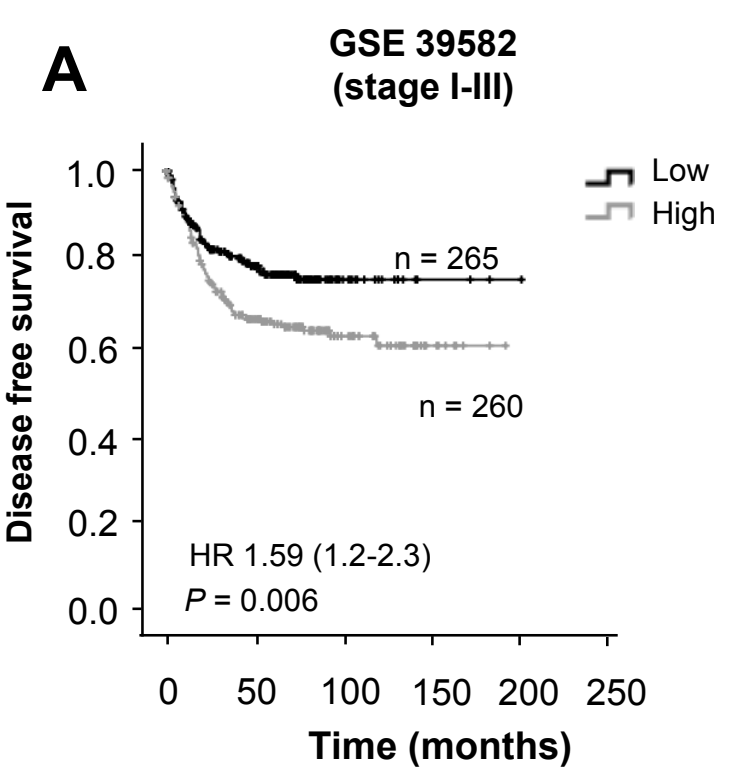

D

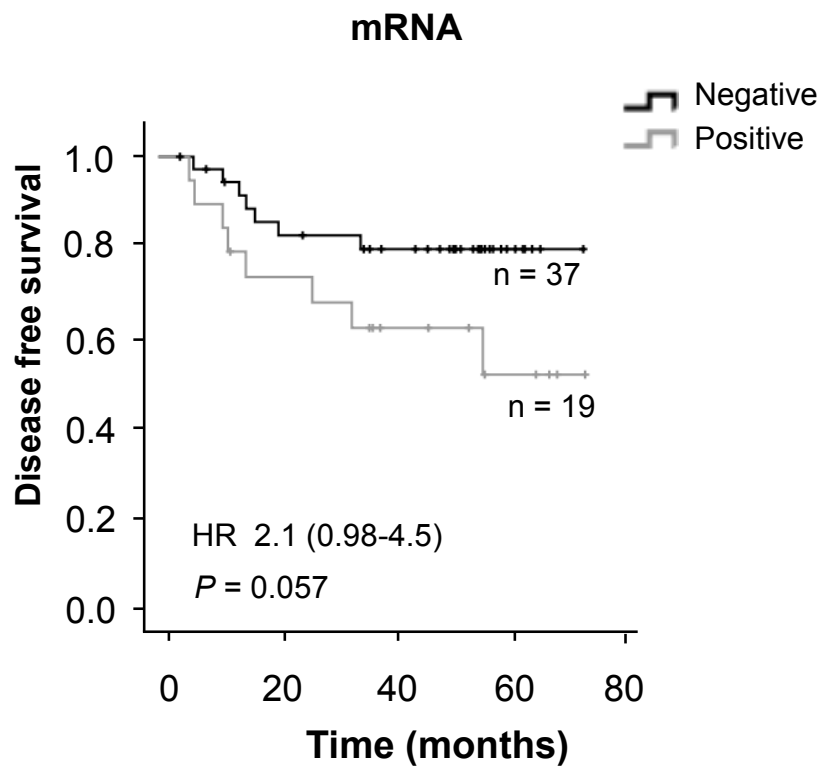

GSE 39582

B (stage I-IV)

C GSE 39582

(stage II)
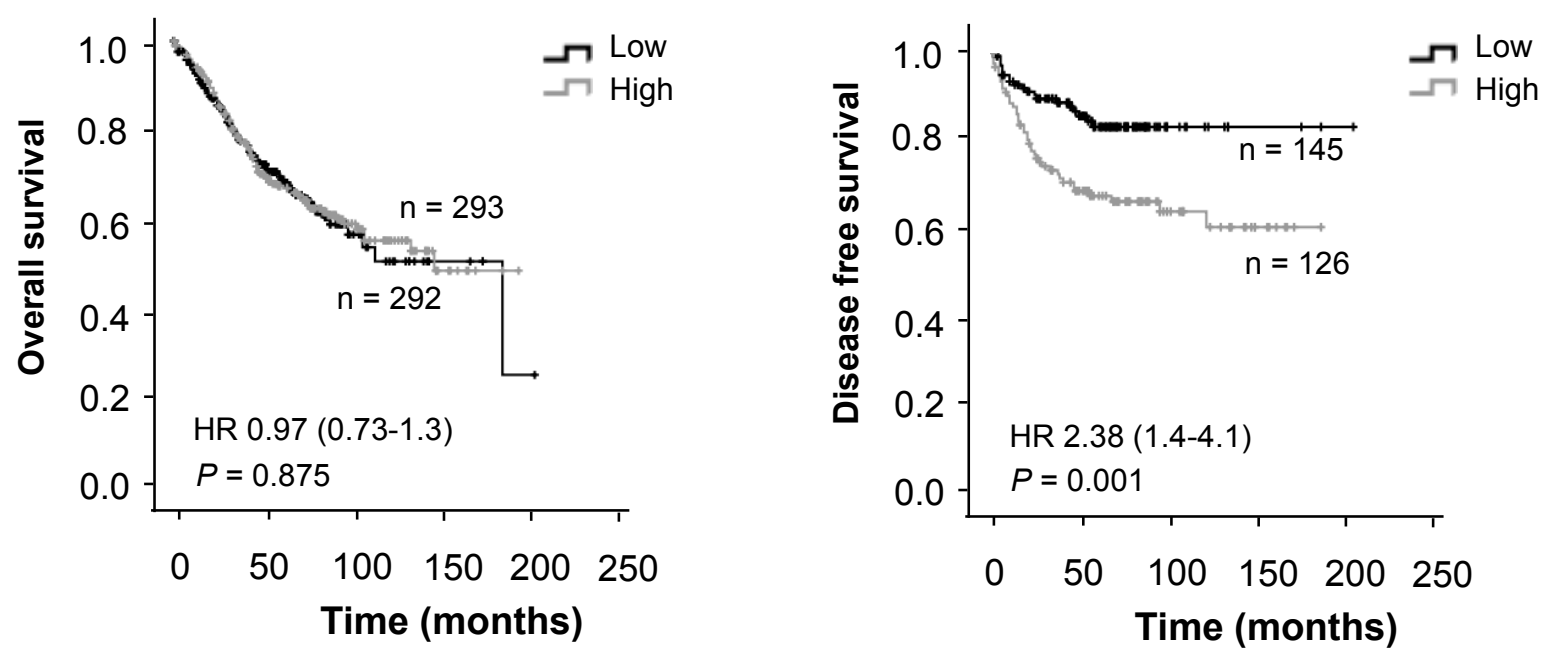

E

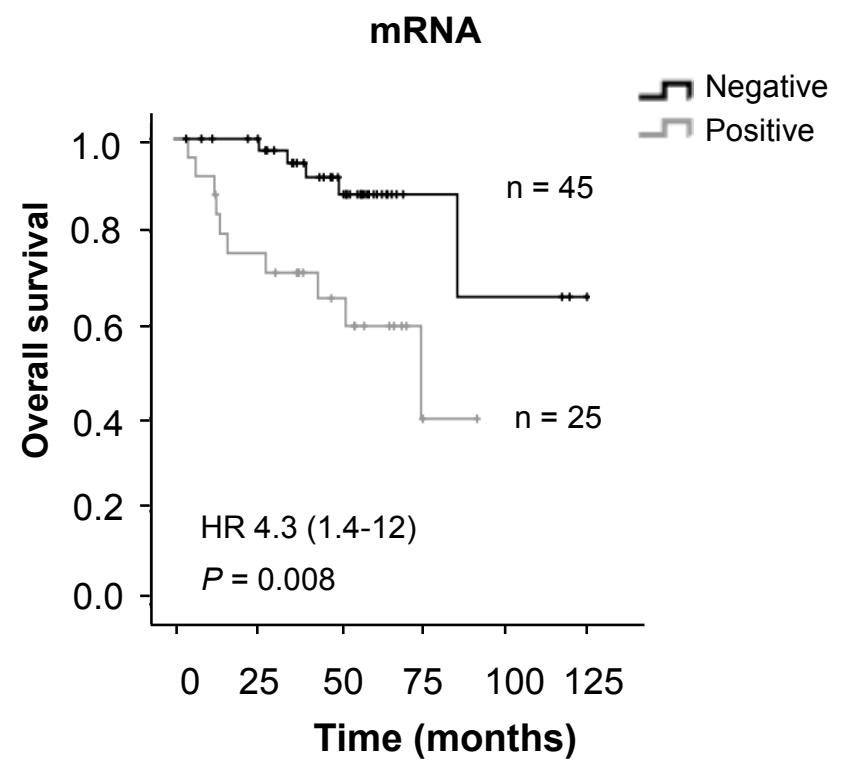

Figure 1 
A

Negative

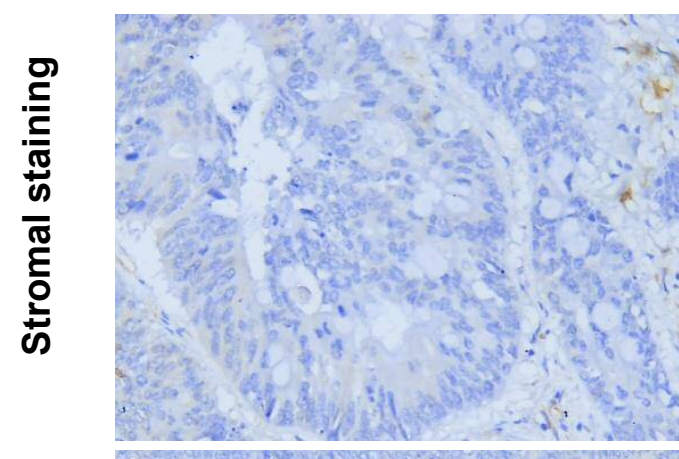

을

B

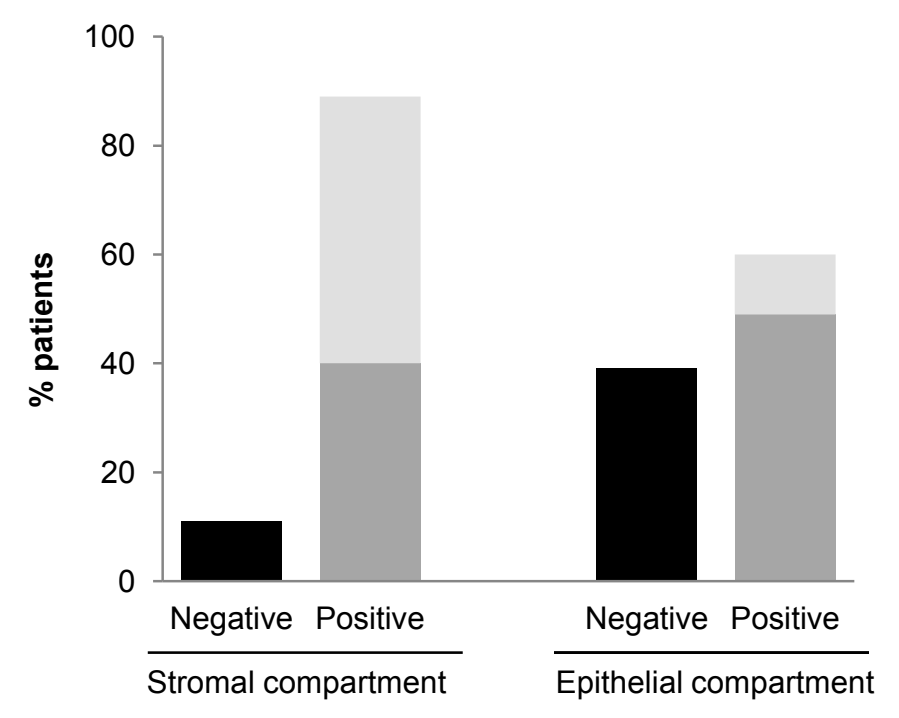

Figure 2

\section{Intense}

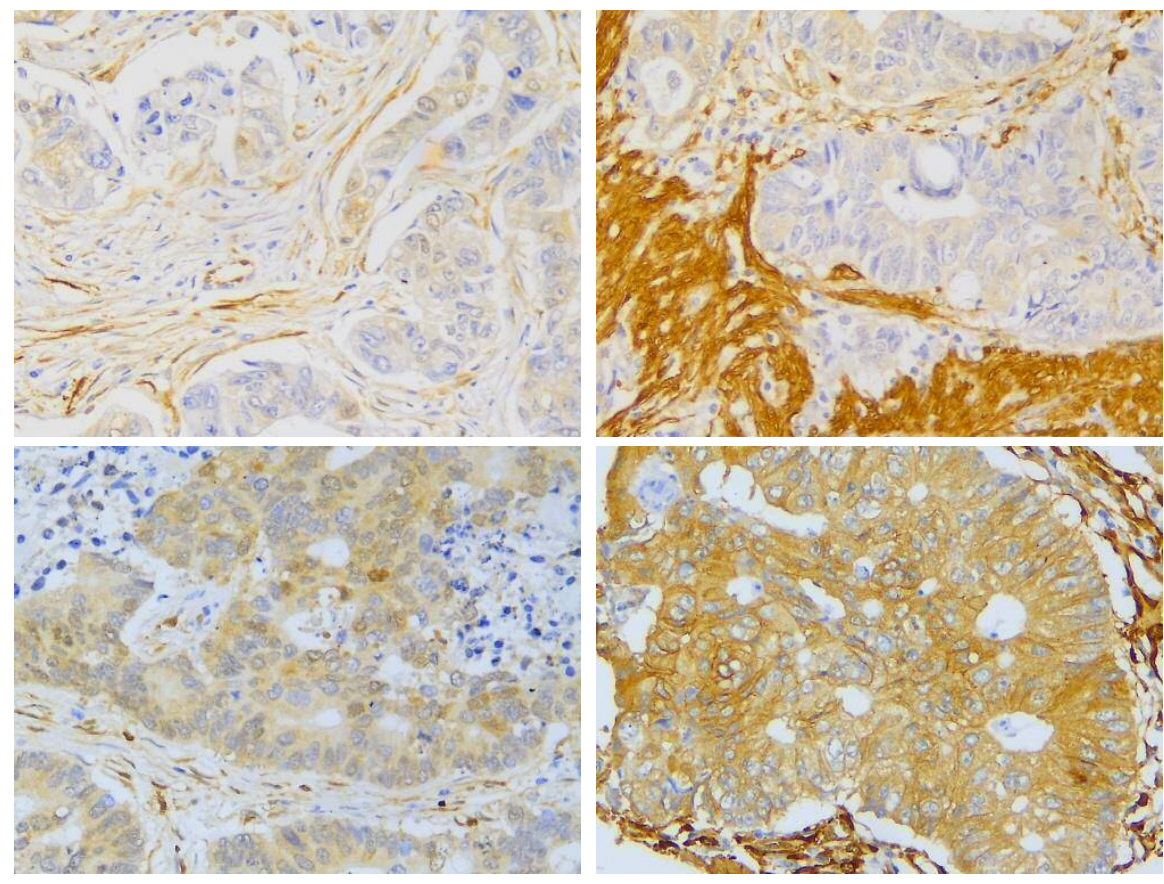

Negative

Moderate

Intense 
A
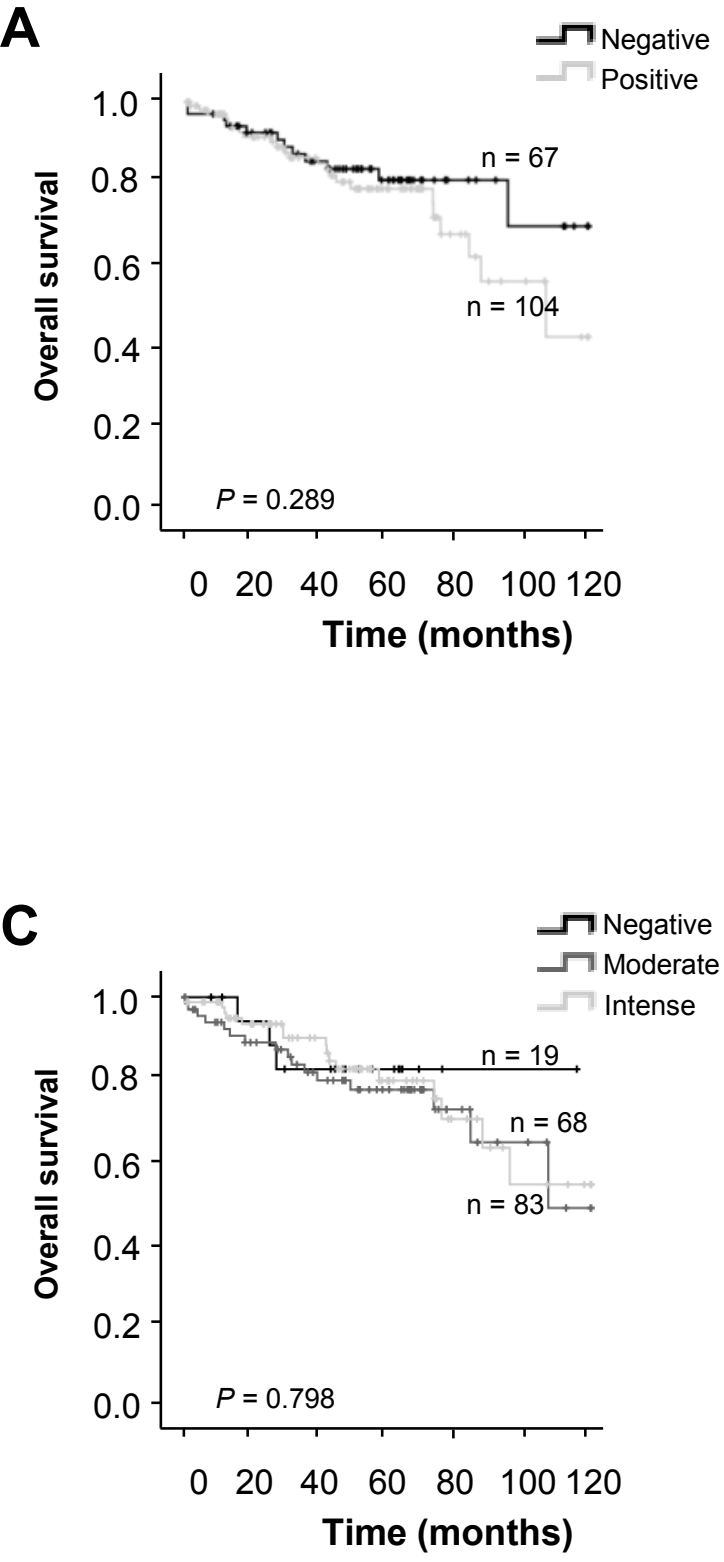

\section{B}
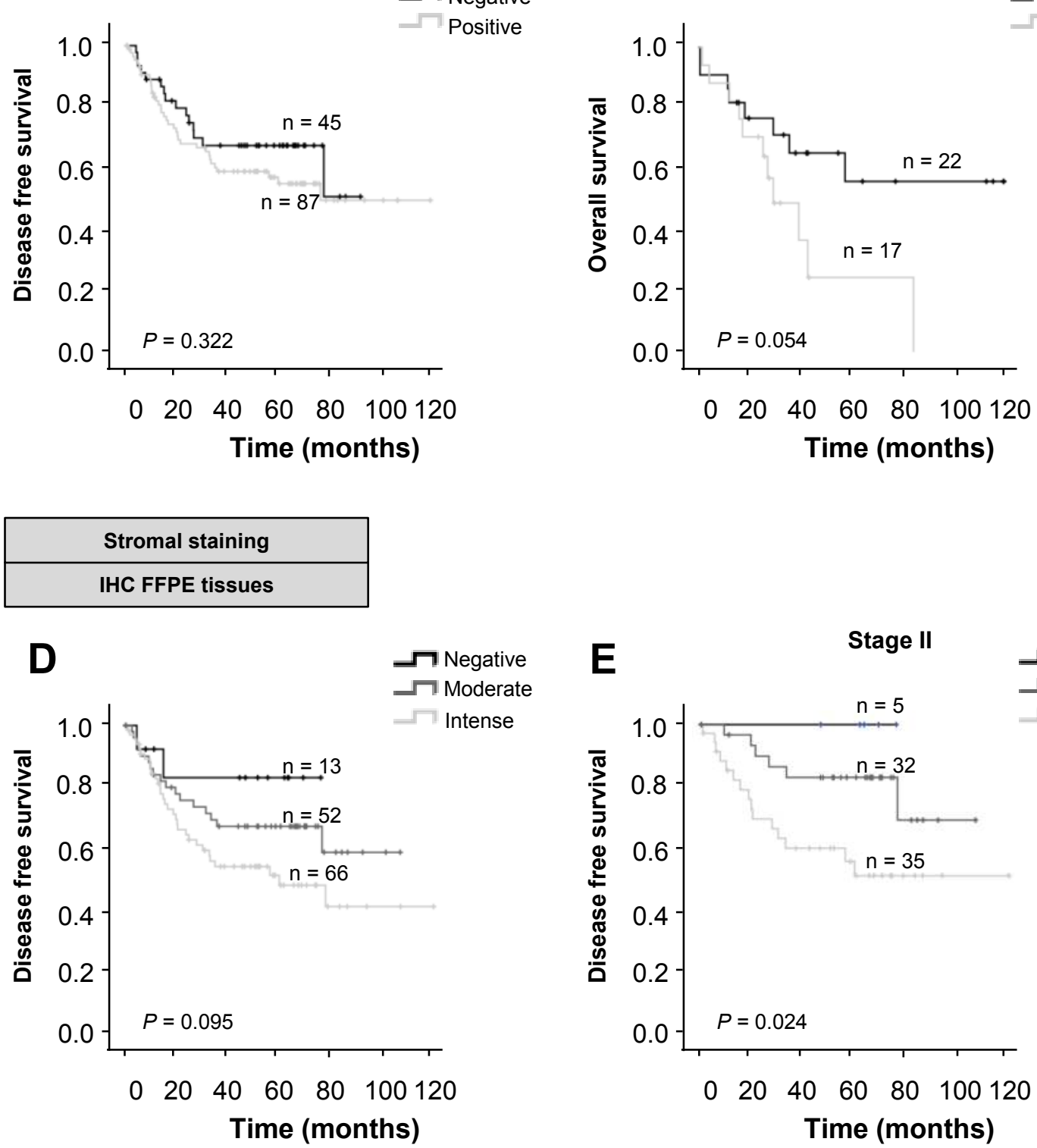

$\Omega$ Negative

F

Stage IV

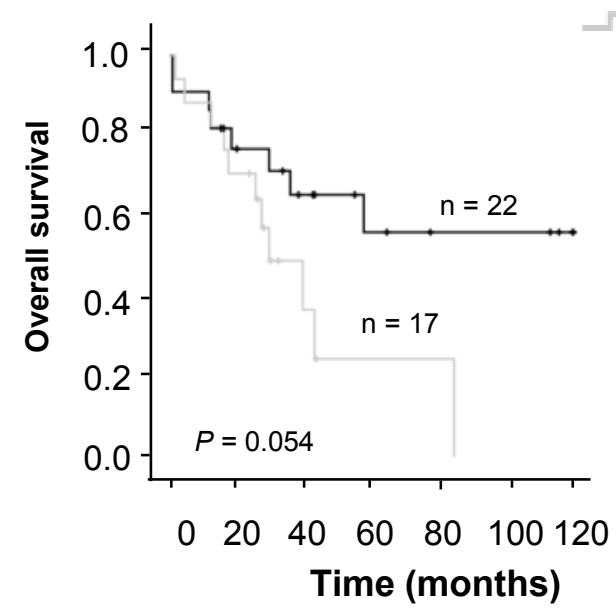

Positive

\section{Figure 3}


A

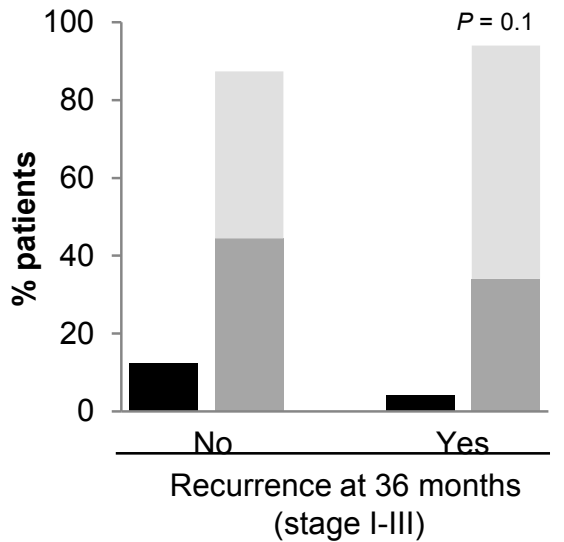

B

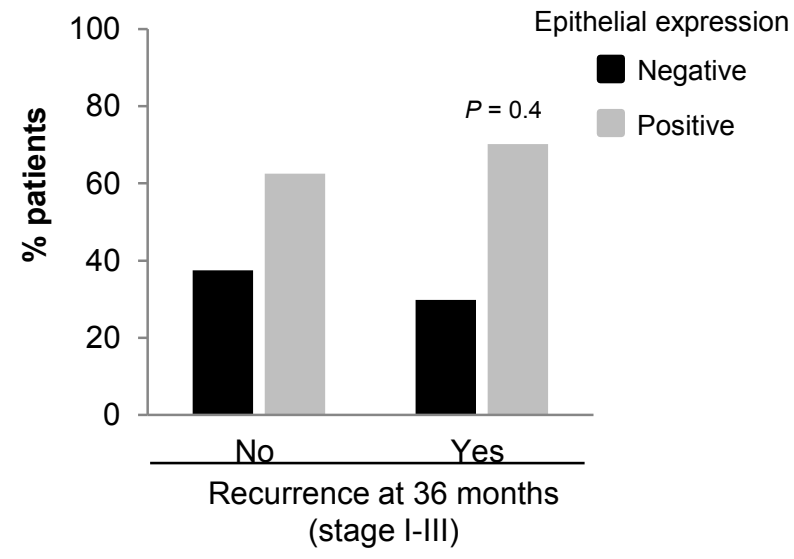

C

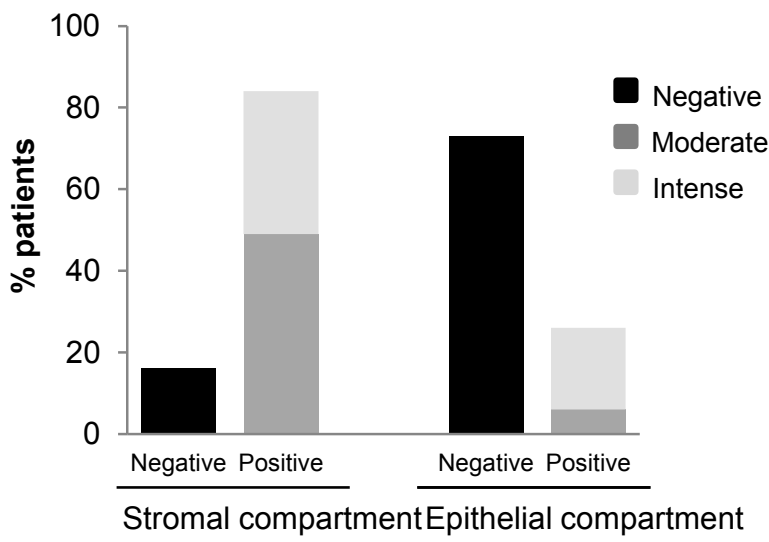

Primary tumor

Liver metastasis

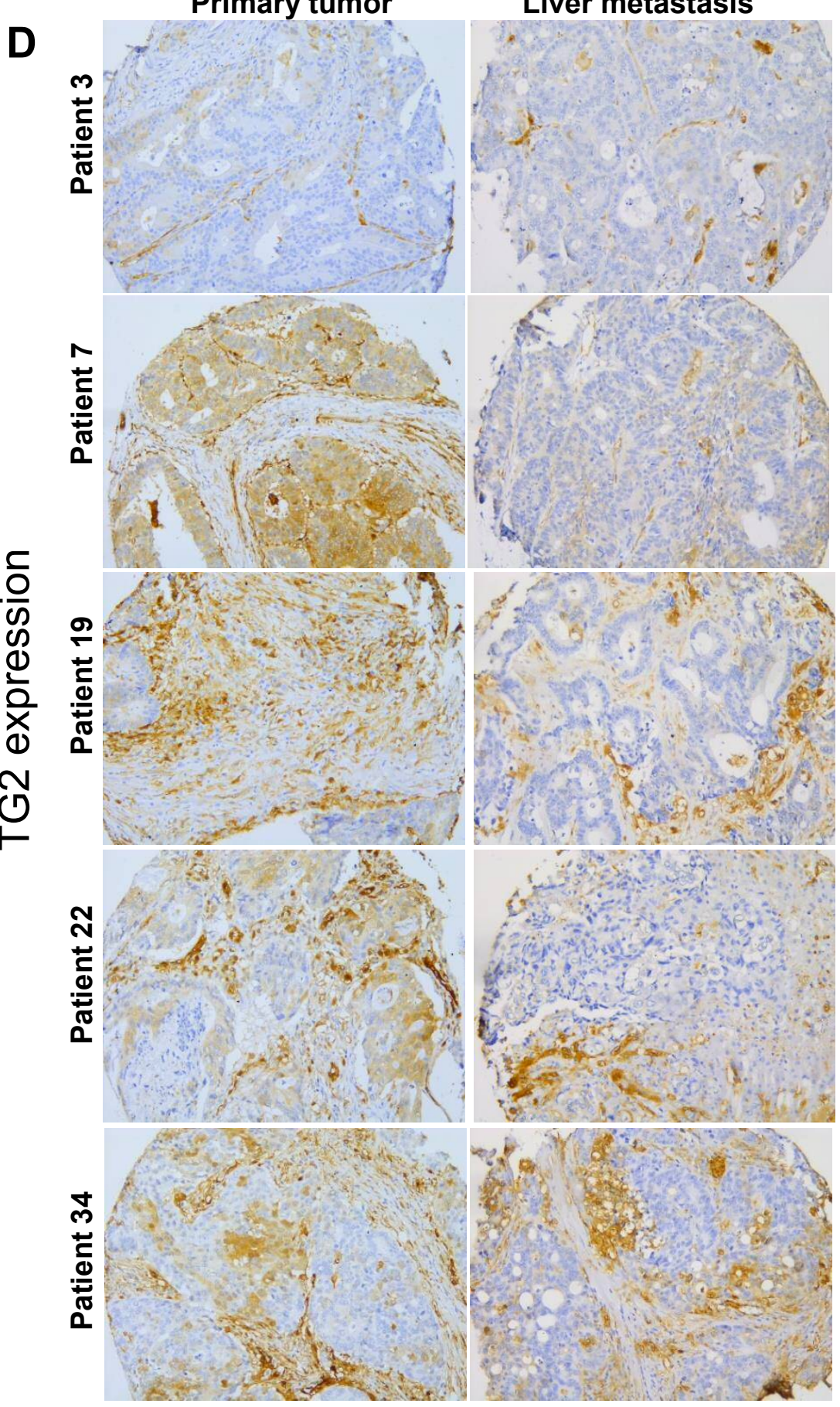

